\title{
Innerstädtische Flusslandschaften als integriertes Handlungsfeld - Planungspraktische Einflussfaktoren der Koordination und Kooperation
}

\author{
Carolin Galler $^{1} \cdot$ Meike Levin-Keitel $^{1}$
}

Eingegangen: 13. Januar 2015 / Angenommen: 31. Dezember 2015 / Online publiziert: 28. Januar 2016

(C) Springer-Verlag Berlin Heidelberg 2016

Zusammenfassung Innerstädtische Flusslandschaften können als Räume verstanden werden, an denen unterschiedliche raumbedeutsame Nutzungen und Belange wie beispielsweise Hochwasserschutz, Naturschutz, Tourismus und Naherholung, Denkmalschutz, Siedlungsentwicklung oder Klimaschutz aufeinanderstoßen. Hier werden integrierte Planungen zur Steuerung der räumlichen Entwicklungen benötigt, für die eine Kooperation von Akteuren verschiedener Verwaltungseinheiten erforderlich ist. Dieser Beitrag widmet sich zunächst den institutionellen Voraussetzungen für kollaborative Planungen im Handlungsraum Flusslandschaften. Dazu werden die Verwaltungsorganisation und die rechtlich verankerten Aufgaben und Planungsinstrumente analysiert. Basierend auf einem planungskulturellen Analyseansatz werden sodann weitere, informelle und eher kulturell geprägte Einflussfaktoren der Zusammenarbeit einbezogen. Am Beispiel der Planungen für die integrierte Entwicklung der Flusslandschaften in Nürnberg und Regensburg werden insbesondere einzelne Charakteristika der Schlüsselorganisationen Wasserwirtschaftsamt und Stadtverwaltung anhand ihrer Handlungsmaximen, ihrer organisationsspezifischen Wahrnehmungen und Bewertungen sowie ihres jeweiligen Selbstverständnisses dargelegt. Es wird deutlich, dass das sogenannte gemeinsame Prozess-Paradigma, also wie die Kooperation im Handlungsfeld innerstädtischer Flusslandschaften von-

Carolin Galler

galler@umwelt.uni-hannover.de

Meike Levin-Keitel

levin@umwelt.uni-hannover.de

Institut für Umweltplanung, Leibniz Universität Hannover, Herrenhäuser Straße 2, 30419 Hannover, Deutschland stattengeht, eine wesentliche Rolle bei der Ausgestaltung der Planungsprozesse und nicht zuletzt auch für die Entwicklung und Gestaltung des Raumes selbst spielt. Es werden Handlungsmöglichkeiten identifiziert, um integrierte Planungsprozesse zu gestalten und zu steuern.

Schlüsselwörter Integrierte räumliche Planung · Wasserwirtschaft · Stadtplanung · Kooperation · Planungskultur

\section{Urban riverscapes as integrated field of planning-coordination and cooperation and the influence of planning practice}

\begin{abstract}
In urban riverscapes different significant land uses and interests come together, for instance flood protection, environmental protection, tourism or local recreation, nature or monument conservation, waterfront development or climate change mitigation. Accordingly, planning for integrated spatial developments is required for which actors of different administrative units need to collaborate. This paper first shows the institutional requirements for a collaborative approach in riverscapes. Therefore the administrative organization and the legally based objectives and planning instruments are analyzed. Based on planning culture as analytical perspective, further informal and more cultural influence factors of cooperation are included. Following the examples of integrated development planning in the riverscapes of Nuremberg and Ratisbon, some interesting characteristics of two key organizations - water management authorities and city administrations - were pointed out by highlighting their guiding principles, their organization-specific perceptions, and validations as well as their particular self-conception.
\end{abstract}


It becomes apparent, that the so called common process paradigm - how the cooperation in urban riverscapes is implemented-plays a crucial role in the way planning processes take place. Last but not least it influences the spatial development and design itself. Relevant parameters for designing and steering integrated planning processes are identified.

Keywords Integrated spatial planning .

Water management $\cdot$ Urban planning $\cdot$ Cooperation $\cdot$

Planning culture

\section{Flusslandschaften als Handlungsraum}

Flüsse und ihre Auen (Flusslandschaften) können als Räume verstanden werden, in welchen eine große Nutzungsvielfalt sowie unterschiedliche Funktions- und Wirkungszusammenhänge aufeinandertreffen. Sie sind komplexe multidimensionale Systeme, die stark von natürlicher Dynamik und (temporär und räumlich) teilweise nur schwer vorhersagbaren Veränderungen geprägt sind, die sich unter dem Einfluss des Klimawandels noch verstärken können. Das charakteristische Landschaftsbild von Flusslandschaften ist darüber hinaus Spiegel regional- und lokaltypischer Nutzungsweisen oder Traditionen und damit bisweilen auch Basis regionaler oder lokaler Identität und Heimat.

Flusslandschaften, und hier speziell innerstädtische Flusslandschaften, werden in diesem Beitrag beispielhaft als
Handlungsräume angesprochen, die einem hohen Nutzungsdruck unterliegen; deshalb sind sie in besonderer Weise geeignet, daran den Bedarf an integrierten (Stadt-) Entwicklungskonzepten aufzuzeigen. Ziel des Beitrags ist es, die Einflussfaktoren für das Zusammenwirken von Akteuren in Planungs- und Umsetzungsprozessen der Stadtentwicklung zusammenhängend darzustellen. Dies impliziert formal-institutionelle Voraussetzungen der Zusammenarbeit ebenso wie informelle Rahmenbedingungen (z. B. kulturelle, situations- und kontextabhängige Variablen). Es werden Möglichkeiten ausgelotet, die integrierte Entwicklung innerstädtischer Flusslandschaften zu gestalten.

Die räumliche Konzentration unterschiedlicher Nutzungen in Flusslandschaften (siehe Tab. 1) kann Katalysator für integrierte Entwicklungen sein und stellt gleichzeitig besondere Anforderungen an die Planung. So haben viele Nutzungen und Raumansprüche eine spezifische Bindung an Flüsse und ihre Auen und sind deswegen nicht oder nur bedingt an alternativen Standorten möglich (z. B. Schifffahrt, Hochwasserschutz, Abwasserentsorgung, Häfen, bestimmte industrielle und gewerbliche Nutzungen). Zudem folgen Verkehrsinfrastrukturen wie Schiene und Straße den Fließgewässern und führen vor allem in städtischen und stadtnahen Bereichen zu Konflikten mit Wohn- und Erholungsnutzungen, für die Gewässer ebenfalls eine hohe Attraktivität aufweisen. Fließgewässer und ihre Auen durchziehen städtische Räume als ein ,grünes Netz“ und bilden Vernetzungskorridore, die gleichermaßen für Erholungs- und Freizeitnutzungen attraktiv sind, wichtige Adern für den Lebensraumverbund darstellen und zudem

Tab. 1 Raumbedeutsame Nutzungen und Ansprüche an Flusslandschaften und Handlungslogiken der Akteure

\begin{tabular}{|c|c|}
\hline Raumbedeutsame Nutzungen und Ansprüche & Perspektive auf die Flusslandschaften und Handlungslogiken der Akteure \\
\hline Hochwasserschutz & $\begin{array}{l}\text { Wasser als potenzielle Gefahrenquelle mit hohem Schadenspotenzial, Schutz vor Hoch- } \\
\text { wasser; Auen als Retentionsraum }\end{array}$ \\
\hline Verkehr (Schifffahrt, Wasserstraßen) & Flüsse als überregionale Verkehrsinfrastruktur \\
\hline Trinkwasserversorgung und Abwasserentsorgung & $\begin{array}{l}\text { Oberflächengewässer als Süßwasserreservoire; Funktionen der Fließgewässer zur Ab- } \\
\text { wasserentsorgung (Reinigungsstufe und Ableitung) }\end{array}$ \\
\hline Energie (Wasserkraftnutzung) & Wasserkraft als alternative Energieform und Baustein der Energiewende \\
\hline Naturschutz und Umweltbelange & $\begin{array}{l}\text { Gewässer und ihre Auen als dynamisches Ökosystem; Hotspots der Biodiversität, Lebens- } \\
\text { räume von (gefährdeten) Arten }\end{array}$ \\
\hline Klimaschutz und Klimaanpassung & $\begin{array}{l}\mathrm{CO}_{2} \text {-Speicher- und Senkenfunktion von Auenstandorten (Kohlenstoff-Bindung durch } \\
\text { Niedermoore und hydromorphe Böden); bioklimatische Funktionen von Flusslandschaften } \\
\text { (Kaltluftentstehungsgebiete und -korridore) }\end{array}$ \\
\hline Freizeit- und Erholungsnutzung und Tourismus & $\begin{array}{l}\text { Gewässer als Anziehungspunkte der Naherholung, in Form von Sport- und Erholungs- } \\
\text { flächen, für Wassersportarten und als Baustein regionaler Grünzugkorridore (,green } \\
\text { corridors“); touristische Nutzungen und Projekte }\end{array}$ \\
\hline Landwirtschaft & $\begin{array}{l}\text { Flusslandschaften als Standorte (fruchtbare Böden) für landwirtschaftliche Nutzung (vor } \\
\text { allem im ländlichen Raum) }\end{array}$ \\
\hline Siedlungsgebiet & $\begin{array}{l}\text { vor allem im städtischen Raum für Siedlungserweiterungen (,,waterfront development“) } \\
\text { oder Revitalisierung innerstädtischer Hafengebiete, weicher Standortfaktor }\end{array}$ \\
\hline Industrie und Gewerbe & $\begin{array}{l}\text { Fließgewässerbenutzung für Kühlung, Prozesswasser und Ableitung; Zulieferungsweg } \\
\text { (Hafen etc.) }\end{array}$ \\
\hline $\begin{array}{l}\text { Denkmalschutz und } \\
\text { Kulturlandschaftsentwicklung }\end{array}$ & $\begin{array}{l}\text { Flusslandschaften und Bauwerke als kulturelles Erbe mit repräsentativer Funktion; Be- } \\
\text { wahrung des lokalen identitätsstiftenden Charakters }\end{array}$ \\
\hline
\end{tabular}


für den Hochwasserschutz von besonderer Bedeutung als Retentionsräume ebenso wie als Gefahrenbereiche sind. Städtische Nutzungen in Auen müssen diese besonderen, durch die Fließgewässerdynamik geprägten Verhältnisse berücksichtigen. Die räumlichen und zeitlichen Wirkungszusammenhänge der vielfältigen raumbedeutsamen Nutzungen und Ansprüche in Flusslandschaften werden von den jeweils auf die eigenen Ziele ausgerichteten Handlungslogiken der Akteure nur bedingt erfasst. Um Konflikte und Nutzungskonkurrenzen zu minimieren sowie Synergieeffekte $\mathrm{zu}$ nutzen und um sektorübergreifende Handlungsstrategien zu entwickeln, sind integrierte Politikund Planungsansätze erforderlich, die ein hohes Maß an Koordination und Kooperation verlangen.

Integrierte europäische Politikansätze, wie sie z. B. mit der EU-Wasserrahmenrichtlinie verfolgt werden, erfordern eine Koordination der Akteure unterschiedlicher Ressorts und Verwaltungsebenen (Moss/Newig 2010; v. Haaren/Galler 2011; Jordan/Schout/Unfried 2013; Köck/Bovet 2015). Koordinationsprobleme, wie unterschiedliche Planungsräume und Betrachtungsmaßstäbe oder gegenseitige Interdependenzen (Moss/Newig 2010), treffen grundsätzlich auch für andere integrierte (Umwelt-) Planungsprozesse zu (z. B. Klimaschutz und Anpassung an den Klimawandel, vgl. Adelle/Russel 2013; Biodiversität, vgl. Paavola/Gouldson/Kluvánková-Oravská 2009), da diese in der Regel ökologisch komplex und sozioökonomisch kontrovers sind (Poch/Comas/Rodríguez-Roda et al. 2004, S. 872). Insbesondere zur Integration unterschiedlicher Umweltbelange (,environmental governance“) (Driessen/ Dieperink/van Laerhoven et al. 2012) muss eine Vielzahl von Akteuren und Akteursgruppen mit unterschiedlichen Handlungslogiken einbezogen und koordiniert werden. Hier sind Governance-Ansätze gefragt, die horizontale, netzwerkartige Beziehungen zwischen öffentlichen und privaten Akteuren berücksichtigen und ein Regieren und Verwalten über unterschiedliche Ebenen hinweg unter Verwendung des gesamten Repertoires an Steuerungsformen (Markt, Hierarchie, Gemeinschaft) ermöglichen (Benz/Dose 2010). Solche Governance-Prozesse finden damit partiell jenseits der hierarchischen Steuerung des Staates statt (Fürst 2001; DiGaetano/Strom 2003; Benz/Lütz/Schimank et al. 2007; Nuissl/Heinrichs 2011).

Polyzentrische und Mehrebenensystemstrukturen („multi-level governance structure“), in denen Hierarchieebenen verschwimmen und Zuständigkeiten sich überlagern, werden zunehmend als geeigneter und resilienter betrachtet (z. B. Huitema/Mostert/Egas et al. 2009; PahlWostl/Lebel/Knieper et al. 2012, S. 27, 32). Dabei handelt es sich um vielfältige Planungs- und Entscheidungsprozesse, wie sie auch zur integrierten Stadtentwicklung in Flusslandschaften stattfinden, die in hohem Maße kontextabhängig und situationsspezifisch sind (Margerum 2008;
Rauschmayer/Berghöfer/Omann et al. 2009) und die, neben fachlich-technischen Determinanten, durch die Integration unterschiedlicher Perspektiven auf die Flusslandschaften geprägt werden (Wiering/Immink 2006, S. 435; Sanon/ Hein/Douven et al. 2012). So werden neben formalen bzw. institutionellen Vorgaben der Kooperation Planungs- und Integrationsprozesse auch planungskulturell, das heißt von kulturellen, individuellen wie gruppenspezifischen Ausprägungen und Interpretationen des Handlungsspielraumes bestimmt (Pahl-Wostl/Lebel/Knieper et al. 2012; LevinKeitel/Sondermann 2014). Jedoch fehlen Informationen und Daten über die Mechanismen der Integration selbst und die Rolle der formalen Rahmenbedingungen sowie der kulturellen, situations- und kontextabhängigen Einflussfaktoren auf die Planungspraxis.

Hier setzt dieser Beitrag an und beantwortet drei Leitfragen: 1) Inwieweit sind (formale) Koordinationsregeln zur ebenen- und sektorübergreifenden Integration als Rahmen der Zusammenarbeit institutionalisiert? 2) Welche (kulturellen) Faktoren beeinflussen darüber hinaus die Koordination und Integration unterschiedlicher Belange und diverser Akteure in der Planungspraxis? Und, als Schlussfolgerung der unterschiedlichen Einflussfaktoren, 3) welche Handlungsmöglichkeiten können identifiziert werden und welche Möglichkeiten bestehen, um integrierte Planungsprozesse zu gestalten und zu steuern?

Im folgenden Kapitel 2 werden der planungskulturelle Analyseansatz als theoretische Perspektive vorgestellt und seine Anwendung in diesem Beitrag erläutert sowie die zwei Fallkommunen und die integrierten Entwicklungen ihrer Flusslandschaften beschrieben. Kapitel 3 zeigt die institutionellen Voraussetzungen für das Zusammenspiel beteiligter Behörden unterschiedlicher Verwaltungsebenen und Ressorts in innerstädtischen Flusslandschaften. Die dazu implementierten formalen Koordinationsregeln der öffentlichen Verwaltung werden beispielhaft anhand des Flussgebietsmanagements in Bayern erläutert. Kapitel 4 zeigt am Beispiel zweier Kommunen (Nürnberg und Regensburg) die unterschiedliche Ausprägung dieser formalen Kooperationsverfahren in der Planungspraxis. Die planungskulturellen Analysen ermöglichen so über die formalen Kooperationsregeln hinaus ein tiefer gehendes Verständnis dieser Prozesse und deren Auswirkungen auf den gesamten Planungs- und Umsetzungsprozess. In Kapitel 5 werden auf der Grundlage der Zusammenführung formeller und informeller Einflussfaktoren Aussagen zur Steuerbarkeit integrierter Planungsprozesse abgeleitet. 


\section{Planungskultur als analytischer Ansatz}

\subsection{Theoretische Perspektive}

Planungskultur als analytischer Ansatz geht nicht von einer guten oder schlechten Planungskultur aus, sondern benennt unterschiedliche kulturelle Einflussfaktoren, die über die formalen institutionellen Rahmenbedingungen hinaus die Ausgestaltung von Planungsprozessen beeinflussen (Fürst 2007; Othengrafen 2012; Reimer 2012; Levin-Keitel 2014). Diese umfassen beispielsweise individuelle wie gruppenspezifische kulturelle Ausprägungen wie Fachsprachen, Selbstverständnisse, Interpretationen des Handlungsrahmens (z. B. in Bezug auf Organisationsformen), diverse Akteurskonstellationen oder situationsspezifische Fenster der Möglichkeiten (siehe theoretische Einordnung des Ansatzes in Levin-Keitel/Sondermann 2014, empirische Einblicke in Levin-Keitel 2015). Einerseits verdeutlicht dies, dass der (planungs-) rechtliche und durch formale Strukturen vorgegebene Rahmen immer einer Interpretation im Rahmen der konkreten Anwendung bedarf und somit nicht von sich aus bereits Garant einer qualitativ hochwertigen Integration aller Belange sein kann. Andererseits veranschaulicht der Planungskultur-Ansatz, wie unterschiedlich die Praxis das Situations- und Kontextspezifische des jeweiligen Ortes mit einbezieht (Friedmann 2005; Othengrafen/Sondermann 2015).

Dieser Artikel basiert auf der Anwendung des Modells von Levin-Keitel 2015 (aufbauend auf Ausführungen in Levin-Keitel/Sondermann 2014). Planungskultur wird hier als ein Zusammenspiel von dem zugrunde liegenden gesellschaftlichen Kontext, kulturellen Artefakten (vor allem Produkte bzw. Ergebnisse räumlicher Planungen und Entwicklungen), Konstellationen und Interaktionen von Akteuren und Akteursgruppen (inklusive ihrer Selbstverständnisse und systemischen Beziehungsgeflechte) und dem planerischen Kontext (wesentliche Strukturen und Regelungen) analysiert. Als tragende Schlüsselvariablen der analytischen Betrachtung wurden folgende Kategorien identifiziert (siehe Levin-Keitel 2015):

- Der Rahmen des Miteinanders, also die Prämissen der Zusammenarbeit (1),

- darauf aufbauend die einzelnen Akteure im Miteinander, also unterschiedliche Organisations-Charakteristika und spezifische Handlungslogiken und Perspektiven (2) sowie

- das miteinander Entstehende, also das gemeinsame Prozess-Paradigma, das sich aus dem jeweiligen Zusammenspiel ergibt (3).

Der (allgemeine) Rahmen des Miteinanders (1) wird anhand einer Analyse der institutionellen Rahmenbedingungen dargestellt (Kapitel 3). Die rechtlichen Grundlagen werden im Hinblick auf den rechtlich verankerten Auftrag zur sektorübergreifenden Abstimmung und Zusammenarbeit beleuchtet. Die (formalen) Koordinationsregeln werden anhand der Aufbau- und Ablauforganisation der öffentlichen Verwaltung herausgestellt. Hier wird deutlich, dass die Kooperation unterschiedlicher Verwaltungen in Bezug auf innerstädtische Flusslandschaften äußerst komplex und eben nicht uniform geregelt ist.

Die Analyse der einzelnen Akteure im Miteinander, also der unterschiedlichen kulturellen Prägungen derinvolvierten Organisationen und ihre spezifischen Handlungslogiken und Perspektiven (2), geht über den Rahmen des Miteinanders hinaus. In vergleichender Perspektive wird hier anhand zweier Fallbeispiele deutlich, dass sich beispielsweise Stadtverwaltungen erheblich voneinander unterscheiden in ihren Handlungsmaximen, ihren Wahrnehmungen und Bewertungen und der Rolle in Kooperationsprozessen. Skizziert werden hier die Organisations-Charakteristika der Wasserwirtschaft und der Stadtverwaltung zweier Fallkommunen, Nürnberg und Regensburg.

Das miteinander Entstehende, also das gemeinsame Prozess-Paradigma (3), das sich aus dem jeweiligen Zusammenspiel ergibt, fokussiert die Analyse der Interaktionen und der Ausgestaltung der Kooperation. Hier geht es darum, wie diese Prozesse der Zusammenarbeit, der Integration unterschiedlicher Belange vonstattengehen: Wie wird die Integration organisiert, welche Verbindlichkeiten werden geschaffen, wie stark ist eine Planungskultur ausgeprägt und was sind schließlich die Eigenschaften dieses gemeinsamen Prozess-Paradigmas (wie werden Schnittstellen gestaltet, welche Lernprozesse finden statt, was ist der Mehrwert)?

Methodisch wird zur Ermittlung des Rahmens des Miteinanders eine Analyse der formalen Kooperationswege durchgeführt, basierend auf einer ausführlichen Literaturanalyse, der Analyse der Internetseiten der beteiligten öffentlichen Stellen und einer Auswertung rechtlicher Grundlagen im Hinblick auf Ziele, Zuständigkeiten und Koordinationsregeln. Diese Ergebnisse werden durch Interviews mit insgesamt 8 Mitarbeitern der staatlichen und kommunalen Umweltverwaltung (Naturschutz, Wasserwirtschaft) und der Raumordnung in Niedersachsen ergänzt und gestützt. Im Rahmen der qualitativen Datenerhebung und -auswertung der zwei Fallbeispiele zu den Organisations-Charakteristika und dem gemeinsamen Prozess-Paradigma werden akteurszentrierte Methoden der Sozialwissenschaften angewandt: Anhand von leitfadengestützten Experteninterviews (Marotzki 2011; Meuser/ Nagel 2011) mit insgesamt 11 Vertretern der Stadtverwaltungen Nürnberg und Regensburg und der Wasserwirtschaftsverwaltung, teilnehmenden Beobachtungen (Lüders 2011) sowie der Analyse von Dokumenten, Protokollen und Beschlüssen des Planungs- und Abstimmungsprozesses 
wird hierzu die empirische Datengrundlage erhoben. Die Auswertung, insbesondere in Bezug auf die Schlüsselkategorien des planungskulturellen Ansatzes, basiert auf Vorgehensweisen und Erkenntnissen der Grounded-TheoryMethodologie (vgl. Corbin 2006). ${ }^{1}$

\subsection{Die Nürnberger und Regensburger Flusslandschaften}

Im Einzelnen werden die Integrationsprozesse zur Erstellung innerstädtischer Entwicklungskonzepte in Nürnberg (Integriertes Stadtentwicklungskonzept „Nürnberg am Wasser“ (Stadt Nürnberg 2012)) und Regensburg (Flussraumkonzept Regensburg (Wasserwirtschaftsamt Regensburg/Stadt Regensburg 2010)) betrachtet. Die beiden Fallbeispiele wurden so ausgewählt, dass sie sich in den sichtbaren Artefakten, also den gebauten städtebaulichen Strukturen, kaum unterscheiden (mittelalterliche Stadtstrukturen, vorliegendes integriertes Gesamtkonzept der Innenstadt) und der formale Rahmen des Miteinanders (vgl. Kapitel 3) der gleiche ist (identischer Verwaltungsaufbau sowie identische bayerische Gesetzgebung).

Fallbeispiel 1 ist das Integrierte Entwicklungskonzept „Nürnberg am Wasser" um die Pegnitz und den Wöhrder See. Im Rahmen des Integrierten Stadtentwicklungskonzeptes wurden in fünf Themenfeldern Leitlinien formuliert sowie Konzeptbausteine entwickelt und strategische Projekte benannt. Das Ergebnis ist ein integrierter Aktionsplan, in dem nicht immer eindeutig räumlich verankerte Maßnahmen dargestellt werden; neben Karten und Plänen spiegelt es sich auch in einem textlichen Teil wider. Laut dem Integrierten Stadtentwicklungskonzept resultieren hieraus „Orientierungen für ein zielgerichtetes Verwaltungshandeln sowie fundierte und belastbare Entscheidungsgrundlagen für die Vorbereitung, Planung, Finanzierung, Förderung und Unterstützung von Projekten und Maßnahmen" (Stadt Nürnberg 2012, S. 11).

Fallbeispiel 2 ist das „Flussraumkonzept Regensburg". Ziel des Flussraumkonzeptes ist es, innerhalb der Stadt Regensburg (entlang der Donau und des Regens) „die vielfältigen Rechtspositionen, verschiedenen Zielsetzungen und Planungen von staatlicher, städtischer und dritter Seite zu analysieren und nach Möglichkeit in Einklang miteinander zu bringen" (Wasserwirtschaftsamt Regensburg/Stadt Regensburg 2010, S. 2). Dabei werden angrenzende Nutzungen einbezogen. Dargestellt werden in dem textlichen Teil des Konzeptes Übersicht und Bestand, Leitlinien und Ziele, Maßnahmen und Umsetzungshin-

\footnotetext{
${ }^{1}$ Die Quellenverweise auf die in den Untersuchungen durchgeführten Experteninterviews sind anonymisiert. Die im Text verwendeten Codes werden mit den zugeordneten Verwaltungseinheiten im Verzeichnis der Interviews (am Ende des Artikels) aufgeführt.
}

weise für einzelne Abschnitte. Im graphischen Teil sind diese räumlich verortet und untereinander abgewogen (vgl. Wasserwirtschaftsamt Regensburg/Stadt Regensburg 2010). Das Regensburger Flussraumkonzept ist aufgrund der gemeinsam gefundenen Detailtiefe in der Darstellung bereits sehr projektorientiert und verdeutlicht die aktive Integration der Flusslandschaften als Handlungsfeld in der Stadtentwicklung.

\section{Der Rahmen des Miteinanders - Institutionelle Voraussetzungen für Planungen im Handlungsraum Flusslandschaften}

\subsection{Verwaltungsorganisation und Planungsinstrumente}

Die öffentlichen Planungs- und Verwaltungsaufgaben sind in Deutschland nach dem Ressortprinzip ${ }^{2}$, das heißt vertikal funktionsbezogen, verteilt (sektoraler Verwaltungsaufbau) (Bogumil/Jann 2009, S. 85 ff.). Für ihre Erfüllung bzw. Umsetzung steht eine Reihe unterschiedlicher Planungsinstrumente zur Verfügung. Zudem bedingt die klassische bürokratische Einlinienorganisation der Ressorts, dass unterschiedliche Aufgabengebiete (wie z. B. Natura 2000, Flussgebietsbewirtschaftung, Hochwasserschutz) weitgehend unabhängig voneinander bearbeitet werden (Bogumil/Jann 2009, S. 154 f.). Auch die Kommunalverwaltungen als Einheitsverwaltungen sind hochgradig arbeitsteilig und hierarchisch organisiert (Bogumil/Jann 2009, S. 158 ff.). Diese eindeutigen Kommunikationsund Kontrollstrukturen führen ebenfalls dazu, dass sich räumlich (teilweise) überlagernde oder wechselseitig beeinflussende Planungen oder Vorhaben in der Regel weitgehend unabhängig voneinander bearbeitet werden. Ferner werden öffentliche (Planungs- und Verwaltungs-) Aufgaben und -Entscheidungen im Mehrebenensystem (EU, Bund, Land, Kommune) bearbeitet und getroffen. Das bedeutet, dass Kompetenzen und Zuständigkeiten innerhalb der einzelnen Ressorts auf unterschiedliche Verwaltungsebenen verteilt sind (z. B. wird Agrarpolitik vor allem auf EU- und Landesebene entschieden, Naturschutz hingegen auf allen Ebenen bis hin zur lokalen (Knüppe/Pahl-Wostl 2013; Nielsen/Frederiksen/Saarikoski et al. 2013, S. 442), Raumplanung auf allen nationalen Ebenen, nicht jedoch auf EU-Ebene). Für die diversen, in städtischen Flusslandschaften relevanten (Umweltfach-) Planungen sind daher unterschiedliche Verwaltungseinheiten auf verschiedenen Planungsebenen zuständig (vgl. Abb. 1 am Beispiel Bayern)

\footnotetext{
${ }^{2}$ Die Ressortzuschnitte sind in den einzelnen Bundesländern individuell geregelt und können sich, insbesondere im Zuge von Regierungswechseln, ändern.
} 


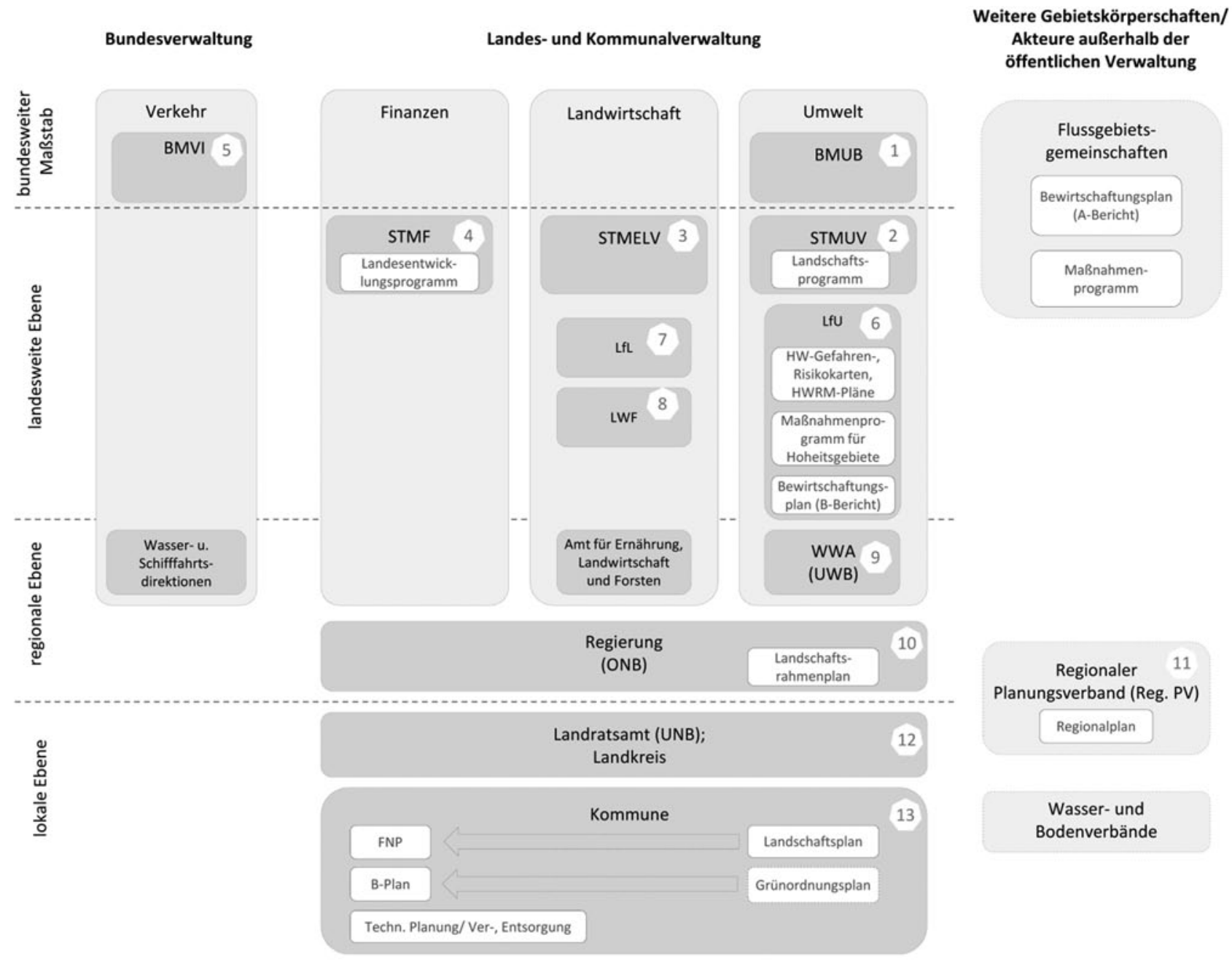

Abb. 1 Verwaltungseinheiten mit Aufgaben im Handlungsfeld Flussgebietsbewirtschaftung und wichtige Akteure außerhalb der öffentlichen Verwaltung (dargestellt am Beispiel Bayern) (Quelle: Eigene Darstellung). Die Aufgabenbeschreibungen sind den Darstellungen auf den Internetseiten der jeweiligen Organisationen entnommen

und die jeweiligen Planungsräume ${ }^{3}$ variieren (,problems of scale“, „problems of fit" und ,problems of interplay“) (Moss/Newig 2010). Dies erschwert die Koordination und Kooperation in Planungs- und Entscheidungsprozessen.

Den (Fach-) Verwaltungen steht auf den verschiedenen Planungsebenen jeweils eine Reihe unterschiedlicher Planungsinstrumente zur Verfügung, die wiederum jeweils

\footnotetext{
${ }^{3}$ Unterschiedliche Planungsebenen: Für Bewirtschaftungsplanung sind in den Bundesländern zumeist obere oder mittlere Landesbehörden oder Landesämter zuständig, während Landschaftsrahmenplanung (als wichtigste Naturschutzfachplanung) von regionalen Planungsverbänden oder Landkreisen erstellt wird. Unterschiedliche Planungsräume: Die Planungsräume der Landschaftsplanung entsprechen den administrativen Grenzen (Landschaftsrahmenplan z. B. für Landkreise), während Bearbeitungsgebiete des Flussgebietsmanagements an naturräumlichen Grenzen (Wasserkörpern) orientiert sind.
}

(http://www.stmuv.bayern.de/umwelt/wasserwirtschaft/wasserwirtschaft_in_bayern/organisation.htm (08.01.15), http://www.lfu.bayern. de/wasser/wrrl/organisation/index.htm (08.01.15), http://www.lfl. bayern.de/iab/duengung/032820/index.php (08.01.15), http://www. wwa-n.bayern.de/ (08.01.15))

auf spezifische Handlungsgegenstände und Ziele ausgerichtet sind. Dazu zählen nutzungsbezogene Fachplanungen (wie z. B. Landwirtschaft und Verkehr) und umweltbezogene Fachplanungen wie Hochwasserschutz und Gewässerbewirtschaftung sowie Landschaftsplanung als umweltmedienübergreifende Fachplanung des Naturschutzes. Die Ziele und Aufgaben sowie Planungsaufträge der in unterschiedlichen Gesetzen verankerten vorsorgeorientierten Umweltplanungen sind nicht deckungsgleich. Zwischen ihnen bestehen Zielkongruenzen und Zielkonkurrenzen (vgl. Grett 2011; Gärditz 2013; Laskowski/ Ziehm 2014; Sanden 2014):

Der Auftrag der wasserwirtschaftlichen Planungen unterscheidet sich von der Naturschutzfachplanung insbesondere dadurch, dass neben den (mit dem Naturschutz weitgehend kongruenten) Umweltzielen auch die 


\begin{tabular}{|c|c|}
\hline \multicolumn{2}{|c|}{ Abkürzungen } \\
\hline B-Plan & Bebauungsplan \\
\hline BMUB & $\begin{array}{l}\text { Bundesministerium für Umwelt, Naturschutz, } \\
\text { Bau und Reaktorsicherheit }\end{array}$ \\
\hline BMVI & $\begin{array}{l}\text { Bundesministerium für Verkehr und } \\
\text { digitale Infrastruktur }\end{array}$ \\
\hline FNP & Flächennutzungsplan \\
\hline HW & Hochwasser \\
\hline LfL & Bayerisches Landesamt für Landwirtschaft \\
\hline LfU & Bayerisches Landesamt für Umweltschutz \\
\hline LWF & $\begin{array}{l}\text { Bayerisches Landesamt für Wald } \\
\text { und Forstwirtschaft }\end{array}$ \\
\hline NGOs & Nichtregierungsorganisationen \\
\hline ONB & $\begin{array}{l}\text { Obere Naturschutzbehörde } \\
\text { (höhere Naturschutzbehörde) }\end{array}$ \\
\hline HWRM & Hochwasserrisikomanagement \\
\hline Reg. PV & Regionaler Planungsverband \\
\hline STMF & $\begin{array}{l}\text { Bayerisches Staatsministerium für Finanzen, } \\
\text { für Landesentwicklung und Heimat }\end{array}$ \\
\hline STMELV & $\begin{array}{l}\text { Bayerisches Staatministerium für } \\
\text { Ernährung, Landwirtschaft und Forsten }\end{array}$ \\
\hline STMUV & $\begin{array}{l}\text { Bayerisches Staatsministerium für Umwelt } \\
\text { und Verbraucherschutz }\end{array}$ \\
\hline UNB & Untere Naturschutzbehörde \\
\hline UWB & Untere Wasserbehörde \\
\hline WWA & Wasserwirtschaftsamt \\
\hline
\end{tabular}

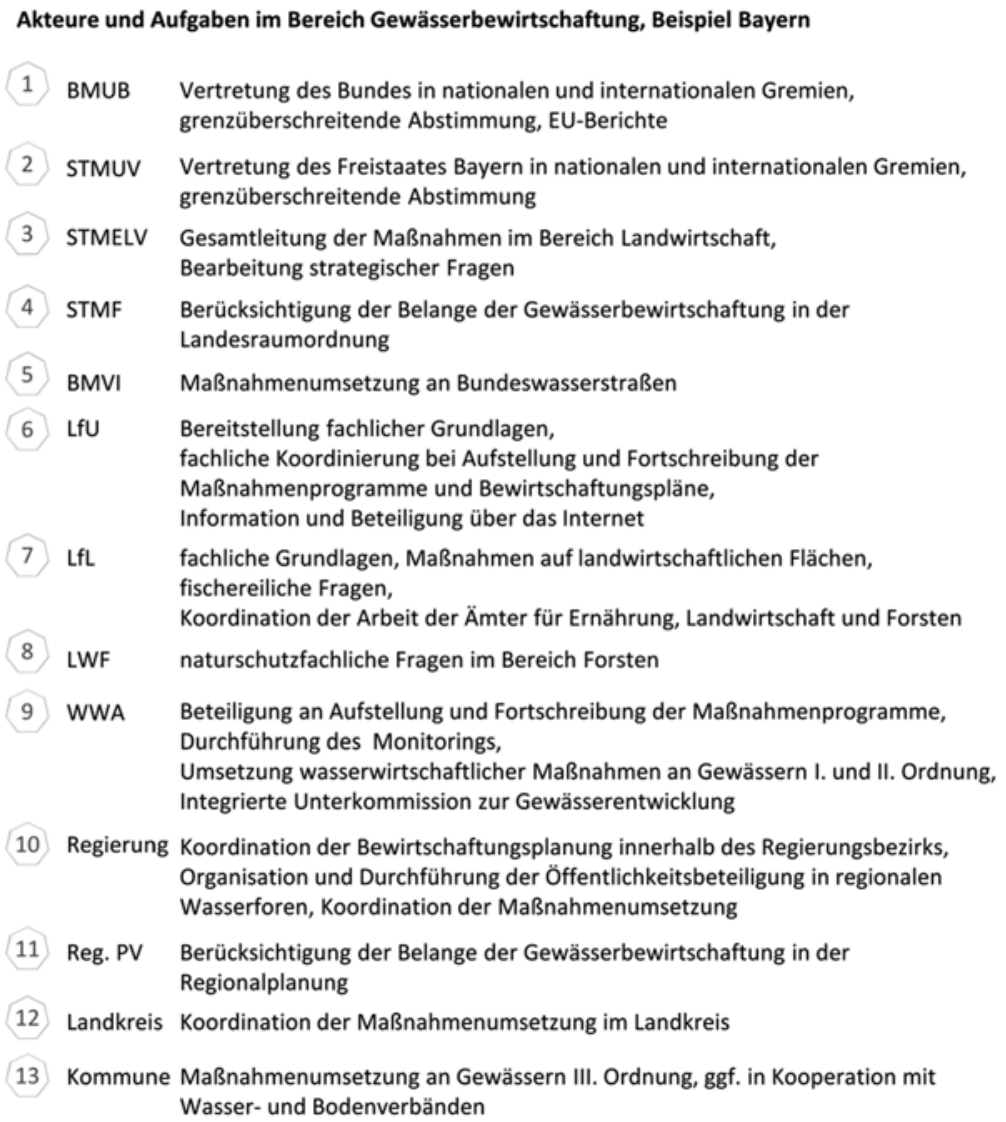

\section{Abb. 1 (Fortsetzung)}

Nutzungsanforderungen (Wasser- und Gewässernutzungen, Hochwasserschutz) berücksichtigt werden müssen. Dadurch erfolgt innerhalb der wasserwirtschaftlichen Planung eine Abwägung der teilweise konfligierenden Schutz- und Nutzungsbelange, sodass die raumbezogenen Zielkonkretisierungen und Maßnahmenvorschläge nicht unbedingt ein naturschutzfachliches und aus Umweltsicht zu favorisierendes Optimum, sondern ein Abwägungskompromiss sind (Reinhardt 2009, S. 517 f.; Gärditz 2013, S. 609).

Die Landschaftsplanung ist in Bezug auf die Ziele, die behandelten Schutzgüter und den Planungsraum eine sehr umfassende Planung: Sie integriert alle Ziele des $§ 1$ Bundesnaturschutzgesetz (und damit die mit diesen kongruenten Ziele anderer Umweltgesetze - insbesondere Wasserhaushaltsgesetz, Bundes-Bodenschutzgesetz). Ihre Aufgabe besteht explizit auch in der umfassenden Gesamtschau der Schutzgüter bzw. Landschaftsfunktionen und darin, diese für den Planungsraum zu konkretisieren und gegebenenfalls gegeneinander abzuwägen bzw. räumliche Schwerpunkte zu setzen. Die Landschaftsplanung ist die Fachplanung des Naturschutzes. Sie erfüllt darüber hinaus auch Aufgaben einer Querschnittsplanung, in dem sie die Erfordernisse und Maßnahmen zur Verwirklichung der Ziele des Natur- schutzes und der Landschaftspflege auch für Planungen und Verfahren anderer Verfahrensträger mit Raum- und Flächenrelevanz aufzeigt (§ 9 (1) Bundesnaturschutzgesetz; vgl. Maaß/Schütte 2014, § 7, Rn. 61). Daher kommt der Landschaftsplanung eine besondere Rolle im System der räumlichen Planung zu, die sie als vorbereitende und informierende Planung einnimmt (Maaß/Schütte 2014, § 7, Rn. 61). Dieser Koordinationsauftrag wird durch Berücksichtigungsgebote unterstützt. So formuliert das Bundesnaturschutzgesetz für alle Bundes- und Landesbehörden den Auftrag, die Ziele des Naturschutzes und der Landschaftspflege im Rahmen ihrer Zuständigkeiten zu unterstützen ( 2 (2) Bundesnaturschutzgesetz), und die Inhalte der Landschaftsplanung sind zu berücksichtigen (§ 9 (5) Bundesnaturschutzgesetz). Der Umwelt- und Naturschutz ist damit eine Querschnittsaufgabe, die auch im Rahmen anderer Planungen und Verwaltungsverfahren umzusetzen ist.

Für einige Umweltbelange sind im jeweiligen Fachrecht keine formalen Planungs- oder Umsetzungsinstrumente verankert, so z. B. für den Bodenschutz. Diese Belange müssen daher von anderen Fachplanungen bzw. der Gesamtplanung mit berücksichtigt bzw. vertreten werden (zum Auftrag der Landschaftsplanung zur Integration von Bodenschutz- 
zielen vgl. Köck 2007). Andere öffentliche Belange, wie z. B. der Klimaschutz, werden nicht in einem eigenen Gesetz geregelt, sind aber über politische Zielsetzungen und Programme oder Konventionen legitimiert oder entsprechende Ziele wurden in andere Gesetze integriert (z. B. Klimaschutz und Klimaanpassung als Grundsatz der Raumordnung gemäß $\S 2$ (2) Nr. 6 Raumordnungsgesetz). Sie sind daher ebenfalls als Querschnittsaufgaben von anderen Fachplanungen bzw., wie in diesem Fall, der räumlichen Gesamtplanung umzusetzen.

Der räumlichen Gesamtplanung kommt eine besondere Funktion zu. Sie hat den Auftrag, die raumrelevanten Belange der verschiedenen Fachplanungen zu koordinieren und abzuwägen (Beckmann/Fürst/Scholles 2004, S. 36 ff.; Fürst 2014). Allerdings sind aufgrund der noch neuen Regelungen zur Umsetzung der EU-Wasserrahmenrichtlinie die Kompetenzen (und damit auch die Koordinationsmechanismen) zwischen Raumordnung (Landesplanung, Regionalplanung) und der wasserwirtschaftlichen Bewirtschaftungsplanung zum Teil noch unklar (vgl. Köck/Bovet 2015, S. 140 ff.). Zu den kommunalen Planungsaufgaben zählen die Bauleitplanung sowie freiwillige (nutzungs- und funktionsspezifische) Planungen und Umsetzungsprojekte im Bereich der Stadtentwicklung. Freiwillige Aufgaben sind z. B. Grünordnungs- und Erholungskonzepte. Die Kommunen als Trägerinnen der Planungshoheit (Art. 28 Abs. 2 Grundgesetz) übernehmen aber auch konkrete fachplanerische Aufgaben: neben der örtlichen Landschaftsplanung, dem Denkmalschutz und der Verkehrsplanung (für Gemeindestraßen) obliegt den Kommunen auch die Umsetzung von Maßnahmen des Naturschutzes und der Flussgebietsbewirtschaftung, wie z. B. die Renaturierung von Fließgewässern. Die Kommunen tragen eine besondere Verantwortung, auch Umwelt- und Naturschutzaspekte in diese Entwicklungskonzepte $\mathrm{zu}$ integrieren $(\S \S 1,1 \mathrm{a}$ Baugesetzbuch; v. Haaren/Galler 2012). Im Zuge der kommunalen Bauleitplanung werden zudem die öffentlichen Belange mit privaten Belangen koordiniert und abgewogen (§ 1 Baugesetzbuch). Die kommunale Bauleitplanung ist bindend für andere öffentliche Behörden (als Flächennutzungsplan gemäß Baugesetzbuch §§ 5-7) und für Privatleute (als Bebauungspläne gemäß Baugesetzbuch $\S \S 8-10$ ), wodurch sie sich von den Fachplanungen unterscheidet.

Trotz der in den Gesetzen verankerten integrierten Ansätze (Raumsauer 2014: § 3, Rn. 52), die Anknüpfungspunkte für entsprechende multifunktionale Entwicklungskonzepte vorgeben, stellen die fragmentierten und sich zugleich zum Teil überlagernden Umweltrechtsbereiche mit konkurrierenden Zielprioritäten eine Herausforderung für die Koordination und eine ganzheitliche Betrachtung der Umweltauswirkungen von Plänen und Projekten dar. So werden die verschiedenen umweltbezogenen Fachplanungen (Landschaftsplanung, Bewirtschaftungsplanung, Hoch- wasserrisikomanagement) - abgesehen von den gesetzlich verankerten Koordinationsregeln - zunächst weitgehend unabhängig voneinander erstellt (Albrecht/Wendler 2009; Hartmann/Driessen 2014; Interview NI_B2). Gärditz (2013, S. 613) bemängelt, dass eine regelungstechnische Verzahnung von Landschaftsplanung und Bewirtschaftungsplanung nicht erfolgt ist. Dies wird nicht zuletzt dadurch verstärkt, dass für die Umsetzung der rechtlichen Vorgaben unterschiedliche Behörden zuständig sind (Ramsauer 2014, $\S 3$, Rn. 50).

Für Planungs- und Entscheidungsprozesse sind daher sowohl eine sektor- und ressortübergreifende wie auch eine vertikale Koordination erforderlich.

\subsection{Strukturen und Regeln zur sektor- und ebenenübergreifenden Koordination}

Für die Koordination von kommunalen Planungen mit der Raumordnung und Fachplanungen der Landesbehörden übernehmen die Bezirksregierungen, die in Bayern „Regierungen“ heißen, als staatliche Mittelbehörden eine zentrale, bündelnde (sektorübergreifend koordinierende) Funktion. Hier laufen die Planungen aus verschiedenen Ressorts zusammen, sodass ein Interessenausgleich (für das Gebiet des Regierungsbezirks) stattfinden kann (Bogumil/ Jann 2009, S. 100). In der Umweltverwaltung sind aufbauorganisatorische Strukturen zur sektorübergreifenden Koordination und Kooperation in unterschiedlichen Formen und auf verschiedenen Verwaltungsebenen eingerichtet. Dazu zählen sowohl Arbeitsgruppen oder Workshops, die von „oben“ (ministeriell) initiiert wurden (Interviews NI B1, NI_B2), wie auch solche, die sich auf Arbeitsebene entwickelt haben (Interviews NI_B1, NI_A1, NI_A2). Der Austausch in diesen zumeist informellen Gremien ergänzt die ablauforganisatorischen Beteiligungsregeln und soll eine Abstimmung und Einigung erleichtern. Neben diesen sich in der Organisationsstruktur niederschlagenden Gremien wird die Zusammenarbeit zwischen verschiedenen Fachbereichen oder Geschäftsbereichen und Fachdisziplinen auch von Einzelpersonen und persönlichen Kontakten von Mitarbeitern unterschiedlicher Bereiche getragen (Interview NI_B1).

Im Rahmen von Planungs- und Verwaltungsverfahren sorgen formale, bürokratische Regeln und Mechanismen für ein Mindestmaß an Koordination. Sie dienen sowohl zur Informationsbeschaffung und -verarbeitung wie auch zur Konfliktlösung und Konsensbildung. Dazu zählen Regelungen zur Mitzeichnung - unter anderem Benehmensund Einvernehmensregelungen (vor allem zur sektoralen Koordination, z. B. gegenseitige Berücksichtigungspflicht von Bewirtschaftungsplanung und Raumplanung) (vgl. Köck/Bovet 2015, S. 139) sowie Prüf-, Genehmigungsund Aufsichtspflichten durch übergeordnete Behörden 
(vertikale Koordination) - oder die Beteiligung im Rahmen von Besprechungen oder Anhörungen (bei größeren Planungsverfahren auch im Rahmen der Beteiligung von Trägern Öffentlicher Belange). Über diese Koordinationsregeln wird auch das (rechtlich verankerte) Gegenstromprinzip (z. B. § 1 (3) Raumordnungsgesetz) umgesetzt. Die Kommunikationsprozesse innerhalb der hierarchisch aufgebauten Sektorverwaltungen bedingen allerdings, dass sektorübergreifende Abstimmungen lange dauern.

Die Anwendung der formellen (Mindest-) Regeln zur Koordination für eine integrierte Planung reicht in der Regel nicht aus (SRU 2004; SRU 2008; Kappet 2006 für den Bereich des Gewässerschutzes). Zudem besteht die Gefahr, dass durch die starke Routinisierung und Spezialisierung der Umweltverwaltung integrierte Lösungen vernachlässigt werden (Jänicke/Volkery 2005). Es haben sich daher weitergehende informelle Koordinations- und Kooperationsformen in Planungs- und Verwaltungsverfahren etabliert und institutionalisiert. Diese informellen Koordinationsund Kooperationsformen zeichnen sich dadurch aus, dass sie freiwillig sind, über das Mindestmaß an formaler Koordination hinausgehen und nicht an enge (formale) Vorgaben (wie z. B. die Form der Unterrichtung, Fristen) gebunden sind. So hat sich in vielen Verfahren (z. B. bei der Aufstellung oder Fortschreibung von Landschafts(rahmen) plänen) eine frühzeitige Beteiligung und prozessbegleitende Einbindung Dritter etabliert (siehe z. B. Bachmann/Hage 2012). Neben einer Koordination bzw. Kooperation von öffentlichen Stellen wird in (Umwelt-) Planungsverfahren häufig auch eine über die formalen Informations- und Beteiligungsregelungen hinausgehende Einbindung nicht öffentlicher bzw. privater Akteure wie Verbände, Unternehmen und der Bürgerschaft angestrebt und umgesetzt (z. B. Runde Tische, Gewässerforen zur Umsetzung der EUWasserrahmenrichtlinie in Niedersachsen).

\subsection{Koordinationsbedarf am Beispiel des Flussgebietsmanagements in Bayern}

Der sektor- und ebenenübergreifende Koordinationsbedarf wird am Beispiel des Flussgebietsmanagements in Bayern deutlich: Zur Umsetzung der flussgebietsbezogenen Gewässerbewirtschaftung gemäß EU-Wasserrahmenrichtlinie wurden Flussgebietsgemeinschaften gebildet, die sich unabhängig von Staats- und Verwaltungsgrenzen an den naturräumlichen Grenzen der Flussgebiete orientieren. Dadurch soll eine flussgebietsweite Koordination von Nutzungen und Maßnahmen umgesetzt werden. Die Zuständigkeiten für Aufgaben zur Umsetzung der EUWasserrahmenrichtlinie (Bestanderfassung, Monitoring, Erstellung von Maßnahmenprogrammen) verbleiben allerdings bei den staatlichen Behörden der verschiedenen
Ebenen, die sich unter dem „Dach“ der Flussgebietsgemeinschaft koordinieren.

Folgende Akteure sind involviert und müssen ihre Aufgaben untereinander abstimmen: Grundsätzlich ist das Umweltressort für wasserwirtschaftliche Aufgaben zuständig. Die planerischen Aufgaben im Zusammenhang mit Bewirtschaftungsplänen und Maßnahmenprogrammen (inklusive der Erfassung und Bereitstellung von Informationsgrundlagen) liegen vor allem beim Landesamt für Umwelt als oberer oder oberster Fachbehörde und beim Wasserwirtschaftsamt als unterer Fachbehörde. Die Zuständigkeiten zur Maßnahmenentwicklung auf landwirtschaftlichen Flächen und im Zusammenhang mit Fischerei liegen hingegen nicht im Umwelt-, sondern im Landwirtschaftsressort und fallen hier in den Aufgabenbereich der Landesämter (vgl. Abb. 1).

Für die Umsetzung von Umweltmaßnahmen entsteht ein Koordinationsbedarf auch dadurch, dass die Umweltfachpläne (insbesondere Maßnahmenprogramme der Wasserwirtschaft, Planwerke der Landschaftsplanung) Maßnahmenvorschläge enthalten, die sich an andere Fachverwaltungen bzw. Flächennutzungen richten und in deren Kompetenzbereich in eigene Fachpläne zu integrieren bzw. umzusetzen sind (z. B. Gewässerschutz über Agrarumweltprogramme). Die Wasserwirtschaft, und das gilt in stärkerem Maße auch für die Naturschutzverwaltung, verfügt nur eingeschränkt über eigene Umsetzungsinstrumente bzw. finanzielle Mittel zur Umsetzung von Maßnahmen ${ }^{4}$ in der Fläche und ist darauf angewiesen, dass Maßnahmen in die Instrumente anderer Fachverwaltungen implementiert werden (v. Haaren/Moss 2011, S. 77 f). Für Maßnahmen in der Fläche (vor allem zum Grundwasserschutz auf landwirtschaftlichen Flächen) sind dies insbesondere Agrarumweltprogramme im Kompetenzbereich des Landwirtschaftsressorts. Daneben ist eine Umsetzung aber auch über Kompensationsmaßnahmen oder -pools möglich, die hauptsächlich im kommunalen Handlungsbereich liegen. Eine Koordinationsfunktion kommt den Bezirksregierungen („Regierungen“) und Landkreisen jeweils für Maßnahmen innerhalb ihres Territoriums zu.

In den kommunalen Zuständigkeitsbereich fällt die Maßnahmenumsetzung an oberirdischen Gewässern III. Ordnung. Auch die Pflege und Entwicklung dieser Gewässer ist eine Aufgabe der Städte und Gemeinden. Allerdings haben die Kommunen die Unterhaltung dieser Gewässer (Gräben und Bäche) in der Regel an Wasser- und Bodenverbände übertragen. Deren Satzung schließt allerdings die Durchführung (Finanzierung) von Entwicklungs-

\footnotetext{
${ }^{4}$ Hier bestehen - mit unterschiedlichen Ausprägungen in den einzelnen Bundesländern - deutliche Unterschiede in der Ausstattung der Ressorts mit Umsetzungsinstrumenten (z. B. „Wasserpfennig“ für Maßnahmen zum Gewässerschutz, Fördermittel im Rahmen von ELER oder Agrarumweltprogrammen) (vgl. v. Haaren/Moss 2011).
} 
maßnahmen im Rahmen der Unterhaltung zumeist aus (Hurck 2011, S. 106). Für größere Seen, Bäche und Flüsse (Gewässer I. und II. Ordnung) sind hingegen die staatlichen Landeswasserbehörden (in Bayern die Wasserwirtschaftsämter) zuständig, für Maßnahmen an Bundeswasserstraßen die Bundesbehörden (Moss 2009, S. 60; Evers/Krause 2010, S. 411 ff.). Für Kommunen an größeren Flüssen (wie in den Fallbeispielen) ist also für die Planung und Umsetzung von Maßnahmen am Gewässer immer eine Abstimmung der kommunalen Planungen mit der Wasserwirtschaftsverwaltung des Landes bzw. der Wasser- und Schifffahrtsverwaltung des Bundes erforderlich.

Unterschiedliche Zuständigkeiten und ein entsprechender Abstimmungsbedarf bestehen auch für Maßnahmen des Hochwasserschutzes oder Maßnahmen, die den Hochwasserschutz tangieren. In der Regel sind Landesbehörden (z. B. in Bayern die Wasserwirtschaftsämter, in Niedersachsen der „Niedersächsische Landesbetrieb für Wasserwirtschaft, Küsten- und Naturschutz") für die Planung und Umsetzung von Hochwasservorsorge- und -schutzeinrichtungen zuständig. Dafür bedarf es aber im Vorfeld der Zustimmung und gegebenenfalls der planerischen Vorbereitung durch die Kommune, die diese Flächen entsprechend bauleitplanerisch ausweisen und sichern muss, um die kommunale Planungshoheit nicht zu verletzen. Für das fertige Bauwerk oder die Maßnahmenfläche ist dann mitunter erneut die Kommune zuständig (z. B. in Bayern; hier wird das Bauwerk nach der Fertigstellung der Kommune übergeben mit 100-jähriger Unterhaltungspflicht).

\section{Planungskulturelle Einflussfaktoren der Kooperation und Koordination in der Planungspraxis}

\subsection{Lokalspezifische Prämissen der Zusammenarbeit abseits rechtlicher Vorgaben}

Die gesetzlich vorgegebenen formalen Regelungen eröffnen Spielräume für eine angepasste Umsetzung. Konkrete Kooperationen und Integrationsprozesse können in der Planungspraxis unterschiedlich ausgestaltet werden. Dies liegt zu einem nicht unerheblichen Teil an den lokalspezifischen Besonderheiten, welche die Ausgestaltung des in Kapitel 2 ausgeführten institutionellen Rahmens der Zusammenarbeit beeinflussen (Aufbau- und Ablauforganisationen). So wirken sich beispielsweise die lokalspezifische Entwicklungshistorie und andere lokale Kontextspezifika auf die Art und Weise des Miteinanders aus.

Das Integrierte Stadtentwicklungskonzept „Nürnberg am Wasser“ (Stadt Nürnberg 2012, S. 10) „formuliert im Ergebnis eines interaktiven Arbeits- und Kommunikations- prozesses eine ressortübergreifende Stadtentwicklungsstrategie für das Gewässernetz der Stadt". Ein Blick auf die Entwicklungshistorie zeigt, dass die etablierten Traditionen und Gewohnheiten, Rituale und Routinen ein wesentlicher Bestandteil der Planungskultur sind. Beispielsweise hat in Nürnberg die integrierte Stadtentwicklung eine sehr lange Tradition. Bereits in den 1980er-Jahren gab es Bestrebungen, ein integriertes Stadtentwicklungsprogramm $\mathrm{zu}$ implementieren, welches aufgrund der starken Topdown-Ausrichtung in den einzelnen Dezernaten jedoch kaum angewandt wurde. Heute ist das Integrierte Stadtentwicklungskonzept eingebettet in den Aufbau einer strategischen Stadtentwicklungsplanung als neues Instrumentarium unter dem Impulsgeber und der Dachmarke „koopstadt - Stadtentwicklung Bremen, Leipzig, Nürnberg", einem Pilotprojekt der Nationalen Stadtentwicklungspolitik. Beteiligt wurden neben Akteuren aus der Zivilgesellschaft hauptsächlich Verwaltungsakteure aller relevanten horizontalen Sektoren sowie vertikaler Beschlussebenen. Ziel der zukünftigen Entwicklung in Bezug auf Gewässer in Nürnberg war ausdrücklich eine Qualifizierung des Umgangs mit Wasser und der bestehenden Nutzungen, die Erlebbarkeit des Wassers in der Stadt zur Erhöhung der Lebensqualität sowie die Entwicklung der Flusslandschaften als Landschaftselement.

Das Flussraumkonzept Regensburg (Wasserwirtschaftsamt Regensburg/Stadt Regensburg 2010) gilt nicht nur in Bezug auf die multifunktionalen Lösungen für die Gestaltung der Regensburger Flusslandschaften als beispielgebend. Der Integrationsprozess zeichnet sich durch eine intensive und langjährige Zusammenarbeit der unterschiedlichen Akteure aus sowie einer optimierten Kombination der verfügbaren Instrumente und Finanzierungsmöglichkeiten. In Regensburg gehört es zur Routine, den aktuellen Stand sowie Probleme, Verzögerungen, Perspektiven monatlich gemeinsam zu diskutieren. Diese etablierten Rituale und Routinen ermöglichen eine einfachere Kommunikation zwischen den Individuen und Akteursgruppen sowie Dritten (z. B. der zu informierenden Öffentlichkeit).

Eine Ausprägung der Entwicklungshistorie können Mythen und Geschichten sein, die sich um die Kooperation ranken. In Regensburg sehr augenfällig ist die Geschichte eines Bürgerprotestes gegen eine Hochwasserschutzmauer. In den 1980er-Jahren gab es massive Proteste gegen den Hochwasserschutz, die letztendlich dazu führten, dass die Hochwasserschutzmaßnahmen in der gesamten Stadt Regensburg zum Erliegen kamen. Erst nach einem kompletten personellen Austausch der Verantwortlichen konnte das Projekt 20 Jahre und einige Flutschäden später wieder aufgenommen werden. Der Prozess baut somit auf der Entwicklung der gemeinsamen Vision in einer langjährigen Tradition der Zusammenarbeit der unterschiedlichen Akteursgruppen auf. 


\subsection{Die einzelnen Akteure im Miteinander, ihre Handlungsmaximen und Selbstverständnisse}

Akteursgruppen wie auch einzelne Akteure können erheblichen Einfluss auf die Ausgestaltung der Zusammenarbeit haben. Hier spielen die organisationsspezifischen Handlungsmaximen, die jeweilige organisationsspezifische Innovationsfähigkeit als auch individuelle und kollektive Wahrnehmungen und Bewertungen eine tragende Rolle. Diese kulturellen Deutungsmöglichkeiten beschreiben Wiering und Immink (2006, S. 435) folgendermaßen: „In contemporary river basin management, decision-making is not only a matter of technical facts: It is also a matter of how the spatial developments in river basins are valued by multiple actors". Um diese Einflussgrößen zu erfassen, wurde das Selbstverständnis der jeweiligen beteiligten Organisationen anhand der Handlungsmaxime der Institution und der organisationsspezifischen Wahrnehmung und Bewertung beispielsweise der Artefakte charakterisiert. Im Folgenden werden die Selbstverständnisse der Wasserwirtschaftsämter und der integriert planenden Stadtverwaltungen (wie Stadtplanungsamt oder Umweltamt) dargestellt.

Die Bayerischen Wasserwirtschaftsämter sind für die Flüsse selbst als auch für die innerstädtischen Flussufer zuständig. Aufgrund der nicht eindeutig geregelten Breite der Gewässerrandstreifen im innerstädtischen Bereich sind der Handlungsraum und die Zuständigkeiten nicht scharf abgrenzbar. Die Wasserwirtschaftsämter agieren als staatliche Verwaltung für die Bereiche der Amtsbezirke auf regionaler Ebene, also über den kommunalen Hoheitsbereich hinaus (vgl. Abb. 1). Ihnen kommen wesentliche Aufgaben im Zuge der Erstellung der großräumig auf die Flussgebiete ausgerichteten Bewirtschaftungspläne und Maßnahmenprogramme zu. Gleichzeitig sind sie mit der Genehmigungs- und Ausführungsplanung konkreter Vorhaben zum Hochwasserschutz oder zur Gewässerentwicklung betraut und setzen diese dann mit eigenen finanziellen und personellen Ressourcen um (vgl. die Kurzdarstellung oben in Kapitel 3). Ihr Auftrag ist es, die Wasserversorgung zu sichern, das Überschwemmungsrisiko zu minimieren und naturnahe Flussauen zu entwickeln (Bayerisches Wassergesetz $\S \S$ 22-23). Die Entwicklungshistorie betrachtend muss man feststellen, dass diese Orientierung in den letzten 30 Jahren einen paradigmatischen Wechsel erfahren hat (Gleick 2000; Molle 2008; Hartmann 2010; Driessen/Dieperink/van Laerhoven et al. 2012). Die (bayerische) Wasserwirtschaft hat sich von einem stark schutzorientierten Ansatz (technische Lösungen für den $100 \%$ igen Schutz) hin zu einem Risikoansatz weiterentwickelt, in dem nicht die Kontrolle über die Umwelt im Vordergrund steht, sondern vielmehr das Leben mit den Risiken, mit unvorhersehbaren Umweltereignissen (z. B. den Folgen des Klimawandels) und veränderten gesellschaftlichen Rahmenbedingungen (Interviews BY_N_W1, BY_R_W3). Zeitgenössische Leitbilder des Wassersektors sind gekennzeichnet von diesem Wechsel von den am technisch Machbaren orientierten ingenieurwissenschaftlichen Lösungen hin $\mathrm{zu}$ einer Koordination und Abwägung gesellschaftlicher Bedürfnisse. Es geht also genauso um Werte und Normen einer Gesellschaft als auch um deren (technische) Umsetzung. Diese Entwicklungen brachten eine sehr moderne, offene und innovationsfreundliche Behörde hervor, die aktiv ist sowohl in räumlich eng abgegrenzten Projektgebieten auf lokaler Ebene bis hin zu Arealen, die nach europäischen Maßstäben definiert sind. Gleichzeitig kann von thematisch fokussierten Experten der Wasserwirtschaftsämter neues Wissen und Erfahrung in diese Ebenen eingebracht werden. Die Interviewpartner der beiden untersuchten Wasserwirtschaftsämter haben eine weitreichende Vision der weiteren Entwicklung der Flusslandschaften (nicht nur) im Stadtgebiet. Diese existiert nicht in Papierform, kann aber als gemeinsame Ausrichtung der Organisation und Wertekanon identifiziert werden.

Anders verhält es sich mit den OrganisationsCharakteristika der Stadtverwaltungen als Einheitsverwaltungen (vgl. die Darstellung in Kapitel 3). Sie sind einer ausgeglichenen ökologischen, ökonomischen und sozialen Entwicklung im Stadtgebiet verpflichtet und am Gemeinwohl orientiert (Baugesetzbuch $\S 1$ (5)). Die Perspektive der Stadtverwaltungen auf die Flusslandschaften richtet sich vom Land auf den Fluss und schließt Landnutzungen und Nutzungen der Wasserzonen mit ihren Ufern oder Flüssen ein. In der Bauleitplanung nehmen die Stadtverwaltungen eine integrative Sichtweise ein, da die Nutzungsansprüche bereits gesammelt, bewertet und untereinander abgewogen wurden. Somit ist die spezifische Sichtweise bereits sehr komplex und in sich abgewogen, jedoch nicht abschließend, da eine Ausgestaltung im Rahmen der Umsetzung erfolgt und die Planung zudem gegebenenfalls an schleichende Veränderungen (wie Klimawandel, demographischer Wandel oder räumlicher Strukturwandel) oder aktuelle Entwicklungen und Anforderungen anzupassen ist. Die Abwägung der Fachbelange innerhalb der Stadtverwaltungen, die in der inneren Organisation in verschiedene Fachabteilungen sektoralisiert ist (vgl. Kapitel 3), wird geprägt von dem politischen Einfluss und den machtpolitischen Konstellationen der Fachabteilungen untereinander und ihren jeweiligen Selbstverständnissen. Die unterschiedlichen Planungsaufträge und Strategien müssen durch interne, informelle, interdisziplinäre Gespräche zusammengeführt werden. Abgesehen von den formellen Instrumenten der Bauleitplanung basiert die Logik der räumlichen Planung und insbesondere der Stadtplanung auf persuasiven Wirkungen, also der verbal-argumentativen Überzeugung der Akteure. Im Unterschied zu den Wasserwirtschaftsämtern setzen die Stadtverwaltungen (Stadt- 
planungsamt oder Umweltamt) einen Großteil der geplanten Maßnahmen nicht selbst um, da sie keine finanziellen Ressourcen hierfür zur Verfügung haben. Die Umsetzung erfolgt vielmehr über die Berücksichtigung bauleitplanerischer Festsetzungen bei der Umsetzung von Projekten, die Implementierung in Fachplanungen oder durch Projekte von Privatinvestoren. Zusammenfassend kann man sagen, dass sich die Stadtplanung zwischen hohen Koordinationsansprüchen, der Bewertung und Abwägung unterschiedlicher Ansprüche und der Gemeinwohlorientierung in einem kulturellen Kontext von mehr als einer Organisation befindet, gepaart mit schwachen Instrumenten und eingeschränkten Umsetzungsmöglichkeiten. Das Selbstverständnis der Stadtverwaltungen hängt sehr stark mit ihren organisationsspezifischen Führungsstilen, den Funktionen und Aufgaben, die sie innehaben und ihrer Entscheidungsfreiheit und ihrem Willen zur Integration zusammen.

\subsection{Das miteinander Entstehende als gemeinsames Prozess-Paradigma}

Was darüber hinaus die Planungskultur der integrierten Entwicklung innerstädtischer Flusslandschaften ausmacht, wird in der Etablierung eines gemeinsamen Prozess-Paradigmas deutlich. Abgesehen von den Prämissen der Zusammenarbeit und der unterschiedlichen (kulturellen) Wahrnehmungen und Bewertungen einzelner Akteure sind deren Zusammenspiel in unterschiedlichen Phasen, mit diversen Outputs und Handlungsroutinen sowie die Ausprägung der gemeinsamen Lernprozesse Kerncharakteristika der Kooperationsprozesse. Die System-Eigenschaften und damit der Mehrwert der Kooperation sind in beiden Fallkommunen sehr unterschiedlich. Während in Regensburg der Output klar im Vordergrund steht, ist in Nürnberg noch der Prozess und die Etablierung eines gemeinsamen Miteinanders ein wichtiger Bestandteil der Ergebnisse.

In Nürnberg hat die integrierte Stadtentwicklung eine lange Tradition, die Pegnitz und der Wöhrder See rückten jedoch erst durch das Stadtentwicklungskonzept „Nürnberg am Wasser" (Stadt Nürnberg 2012) in das Bewusstsein. Für die Prozessorganisation bedeutet dies, dass hier wenig auf Traditionen und Routinen zurückgegriffen werden kann und es in der beginnenden Kooperation sehr viel mehr auf gemeinsame Mentalisierungsprozesse ankommt, beispielsweise gemeinsam eine Vorstellung von Nürnbergs Entwicklung am Wasser zu erarbeiten, die unterschiedlichen Überzeugungen, Einstellungen und Wünsche zu erkennen und $\mathrm{zu}$ akzeptieren oder die Eigenheiten und Besonderheiten in Nürnberg $\mathrm{zu}$ integrieren, um der Kooperation eine Organisation $\mathrm{zu}$ geben und diese gemeinsam $\mathrm{zu}$ gestalten. Hier wird auch die relativ schwach ausgebildete gemeinsame Planungskultur unter den Beteiligten deutlich.
Die Kooperation ähnelt noch keinem ,eingespielten Team“ (Interview BY_N_S2), wo einer die Belange des anderen kennt und gleich mitdenken kann. Diese Umsicht und gemeinsame Perspektive auf den Prozess ist weniger stark ausgebildet und wird noch etabliert. Dies wird besonders an den Schnittstellen zwischen Fachabteilungen der Stadtverwaltung und der Wasserwirtschaft deutlich: Zwischen beispielsweise dem Umweltamt und der Wasserwirtschaft sind die Kooperationen sehr routiniert organisiert, während insbesondere die stadtverwaltungsinternen Schnittstellen weniger ausgebildet sind. Lernprozesse sind die Grundlage für ein zukünftiges vertrauensvolles Miteinander, indem Fenster der Möglichkeiten geschaffen und auch gesehen werden, indem einzelne Vertreter unterschiedlicher Fachplanungen beginnen, miteinander zu planen und gemeinsame Routinen und Traditionen der Zusammenarbeit etablieren können. Somit war es für einige Beteiligte ein ,aufwendiger und sehr kommunikativer Prozess [...]. Der hat vieles in der Verwaltung auf den Kopf gestellt, was Terminplanung angeht, was Interkommunikation angeht, und Zusammenarbeit, war aber sehr, sehr lehrreich“ (Interview BY_N_S3).

Die integrierte Entwicklung der Regensburger Flusslandschaften ist bereits seit Jahren engmaschig organisiert und geplant und befördert eine Vielzahl an Handlungsroutinen auf unterschiedlichen Ebenen (von der Koordinierungsgruppe bis zur Projektgruppe). Insgesamt ist die gemeinsame Planungskultur oder das gemeinsame Prozess-Paradigma sehr stark ausgeprägt. Es gibt eine enge Verbindung der einzelnen Akteure, auch wenn diese nicht immer einer Meinung sind. „Aber unterm Strich ist es wichtiger, in einer Stadt, und gerade in einer Stadt der Größe Regensburgs, einen stadtgesellschaftlichen Zusammenhang herzustellen. Das muss kein Konsens sein, sondern man muss gesprächsfähig miteinander sein" (Interview BY_R_S1). Dies manifestiert sich in den Eigenschaften des gemeinsamen Prozess-Paradigmas, wie der Ausgestaltung der Schnittstellen im System (beispielsweise, indem nur einvernehmliche Lösungen weiterverfolgt werden, oder der gemeinsamen „Bürgerinformationschoreographie“ (Interview BY_R_S2)), den gemeinsamen Lernprozessen, die initiiert und gefördert werden (beispielsweise Schlussfolgerungen aus der Vergangenheit, Kurskorrekturen im Verlauf) oder dem entstehenden Mehrwert der Kooperation. Der Mehrwert wird von den Akteuren benannt, wenn sie von der integrierten Entwicklung als „Maßnahmenlokomotive“ (Interview BY_R_S1) sprechen, wenn Akzeptanz und Vertrauen in die gemeinsame Arbeit wertgeschätzt werden oder von der ,wissenden Gelassenheit“ (Interview BY_R_W2) als Grundprämisse reden, denn es ist „manchmal eben besser, nicht alles durchzuplanen, sondern Dinge wachsen zu lassen“(Interview BY_R_W2). 


\section{Zur Steuerbarkeit integrierter Planungen im Handlungsfeld (innerstädtischer) Flusslandschaften}

Der Auftrag zu einem abgestimmten Vorgehen in Planung und Umsetzung ist insbesondere durch inhaltliche Verschränkungen und Zielkonvergenzen in den Umweltrechtsbereichen, durch Berücksichtigungsgebote sowie durch ablauforganisatorische, formelle wie informelle Koordinationsregeln institutionell verankert. Es wurde deutlich, dass eine Analyse der institutionalisierten Regeln jedoch nicht ausreicht, um die vielfältigen (integrierten) Planungsprozesse mit ihren Koordinations- und Kooperationsmechanismen zu verstehen. Vielmehr bestimmen kulturelle und kontextspezifische Aspekte wie die Interpretation des Handlungsrahmens und die Selbstverständnisse der Akteursgruppen die Ausgestaltung der Planungsprozesse und die langfristige Implementierung gemeinsamer Ziele. Diese unterschiedlichen Einflussfaktoren, die in den vorangegangenen Kapiteln weitgehend isoliert analysiert und dargestellt wurden, können bei der Suche nach praktischen Ansatzpunkten für die Steuerung integrierter Entwicklungsprozesse nicht getrennt voneinander , bedient' werden. Vielmehr beeinflussen sie sich gegenseitig und bestimmen in ihrem Zusammenwirken die Ausgestaltung integrierter Planungen. Vor diesem Hintergrund stellt sich die Frage, inwiefern die Koordination und Kooperation zwischen Akteuren unterschiedlicher Verwaltungsebenen und Ressorts steuerbar sind bzw. bis zu welchem Grad die identifizierten Einflussfaktoren selbst wiederum beeinflussbar sind und Ansatzpunkte für die Justierung von integrierten Entwicklungs- und Umsetzungskonzepten sein können. Mögliche Einflussfaktoren und Ansatzpunkte für die Steuerung einer integrierten Entwicklung werden in folgenden Bereichen gesehen:

1. Umgang mit formalen Koordinationsregeln

2. Wechselwirkungen zwischen Kompetenzen und Kooperationsformen

3. Fachkulturen und Selbstverständnisse verstehen und achten

4. Strategische Überlegungen im Zusammenspiel mehrerer Fachdisziplinen analysieren und Win-win-Situationen schaffen

5. Situationsspezifität und Kontextabhängigkeit erkennen

(1): Insbesondere wenn die Erstellung formaler Pläne betroffen ist, müssen die institutionalisierten Mindestvorgaben zur Koordination (wie Informations- und Beteiligungspflichten, Fristen für Stellungnahmen) im Planungsprozess beachtet werden. Darüber hinaus sind auch Berücksichtigungspflichten, insbesondere von Inhalten der Landschaftsplanung, anzuwenden und geeignete Inhalte sind in die jeweiligen Planungen zu integrieren. In der Praxis findet diese Berücksichtigung allerdings nicht immer statt (Interview NI_A1). Um die Abstimmung und Zusammenarbeit der Akteure darüber hinaus zu fördern, ist es nicht zielführend, weitere Koordinations- und Kooperationsregeln rechtlich zu verankern. Das würde der Unterschiedlichkeit und Situationsspezifik der Planungen nicht gerecht. Mitunter können stark formalisierte Informations- oder Beteiligungsregeln kontraproduktiv wirken und eine Kooperation hemmen.

(2): Die Stellung einer Behörde im vertikalen und horizontalen Verwaltungsaufbau sowie ihre (formalen) Zuständigkeiten und Kompetenzen wirken immer auch in informelle Planungsprozesse hinein. Dies ist entscheidend dafür, wer in den Planungsprozess zur integrierten Entwicklung innerstädtischer Flusslandschaften eingebunden wird. Das Wissen um die Kompetenzen der federführenden und beteiligten Akteure (z. B. Weisungs-, Aufsichtsrechte) beeinflusst den Kooperationsprozess, auch wenn diese in dem integrierten Planungsprozess nicht direkt angewandt werden (,Schatten“ der Hierarchie). In den Interviews wurde deutlich, dass die Akteure der öffentlichen Verwaltung bestrebt sind, ihre Kompetenzen, die ihnen aufgrund ihrer Stellung im hierarchischen Verwaltungssystem zukommen, vollumfänglich zu nutzen und ihren Entscheidungsspielraum zu erhalten. Dazu werden klare (bürokratische) Kompetenzregelungen als hilfreich erachtet, während Kooperationen diese Grenzen verwischen und gegebenenfalls bedeuten, auf das Ausschöpfen des eigenen Handlungsspielraums zu verzichten (vgl. auch Fürst 2014, S. 452). Wenn es gelingt, den beteiligten Akteuren den Mehrwert eines integrierten Konzeptes für das gemeinsame Handeln vor Augen zu führen, wächst die Bereitschaft, Autonomie zugunsten der Kooperation bzw. „systemischer Führung" aufzugeben (Fürst 2014, S. 453, 455).

(3): Die federführenden Akteure oder Organisationseinheiten müssen sich nicht nur der Kompetenzverteilungen in der formalen Aufbau- und Ablauforganisation bewusst sein, sondern sich auch die jeweiligen Selbstverständnisse der Verwaltungseinheiten deutlich machen. Die Handlungslogiken der Akteure werden neben dem gesetzlichen Auftrag wesentlich von Intentionen, Interessen und der Wahrnehmung der Flusslandschaften im Kontext der eigenen Handlungsoptionen und ihrer Interpretation bestimmt. Eine wesentliche, wenn auch nur bedingt steuerbare Einflussgröße wird in „interkulturellem“ Wissen und entsprechenden Erfahrungen der Akteure gesehen, die ein gegenseitiges Verständnis und den Umgang mit verschiedenen Fachsprachen fördern können. Diese ,interkulturellen" Kompetenzen können durch interdisziplinären Austausch erworben werden. Dies bestätigen Befragte aus den Fachverwaltungen Naturschutz und Wasserwirtschaft. Ein gegenseitiges Verständnis für die jeweils anderen Fachbelange in Wasserwirtschaft und Naturschutz sei vorhanden 
und Basis für Abstimmungs- und Kooperationsprozesse und eine stärker integrierte Umweltplanung. Dies hänge nicht zuletzt auch mit einer zunehmend interdisziplinären Zusammensetzung der Wasserwirtschaftsverwaltung und einer stärkeren ökologischen Ausrichtung im Zuge der EU-Wasserrahmenrichtlinie zusammen (Interview NI_B1). Andererseits seien die ökologischen Zusammenhänge und Bewertungsmethoden sehr komplex und nur begrenzt an „Nicht-Fachleute“ vermittelbar (Interview NI_B2).

(4): Zudem spielen strategische Überlegungen im Zusammenspiel von mehreren Fachdisziplinen und Sektorverwaltungen und unterschiedliche Fachkulturen eine Rolle. Zum Beispiel wird eine frühzeitige Integration der Umweltbelange von Mitarbeitern der Naturschutzverwaltung nicht immer angestrebt, um auch die ,unabgewogenen“ Naturschutzbelange für den politischen Entscheidungsprozess und für die Öffentlichkeit darstellen zu können. Dies wird auch von Vertretern der räumlichen Gesamtplanung als wichtig erachtet, um Transparenz über die Umweltbeeinträchtigungen von Planungen und Vorhaben herzustellen (Interviews NI_C1, NI_A2).

(5): Sowohl in den Interviews als auch in den untersuchten Fallbeispielen wurde das hohe Maß an Situationsspezifik und Kontextabhängigkeit deutlich. Koordinations- und Kooperationsprozesse verlaufen in Abhängigkeit von ihrer Entwicklungshistorie und den jeweiligen spezifischen Akteurskonstellationen mit ihren unterschiedlichen Handlungslogiken, wobei Rahmenbedingungen unterschiedlich wahrgenommen und interpretiert werden. Daher ist die gesamte Prozesskoordination mitzudenken und Hindernisse und Chancen sind zu identifizieren. Welche Rollen von den Beteiligten im Planungsprozess übernommen werden (können) (z. B. Federführung, Moderation, Mediation), ist nicht allein anhand des Verwaltungsaufbaus oder der fachlichen Federführung ersichtlich, sondern wird erst im situationsspezifischen System offenbar (systemische Betrachtungsweise). Bestimmte öffentliche Stellen können im Planungsprozess aufgrund ihrer Aufgaben und Kompetenzen eine Schlüsselfunktion für das Gelingen des Integrationsprozesses übernehmen. In den Fallbeispielen kam den Wasserwirtschaftsämtern eine solche Schlüsselfunktion zu. Der Aufgabenbereich der bayerischen Wasserwirtschaftsämter umfasst sowohl die Planung und Umsetzung wie auch einen Beratungsauftrag der Kommunen und Träger Öffentlicher Belange für wasserwirtschaftliche Belange. Dadurch kennt das Wasserwirtschaftsamt als unteres Landesamt die lokalen Probleme und kann in Planungsprozessen eine Vermittlerrolle zwischen den (oberen) Landesbehörden und den Kommunen einnehmen. Für die horizontale, sektorübergreifende Abstimmung übernehmen die Wasserwirtschaftsämter insofern eine vermittelnde Rolle, als sie selbst interdisziplinär aufgestellt sind und zumindest teilweise sowohl Gewässer- schutz, Naturschutz und Belange der Wassernutzung und des Hochwasserschutzes von vornherein koordinieren.

Die betrachteten Fallbeispiele bilden nur einen kleinen Ausschnitt der Bandbreite möglicher integrierter Planungsprozesse und Entwicklungskonzepte in städtischen Flusslandschaften ab. Die aufgezeigten Einflussfaktoren und Ansatzpunkte sind daher nicht abschließend zu verstehen und sollten durch weitere empirische Untersuchungen verdeutlicht, differenziert und erweitert werden. Weiterer Forschungsbedarf wird daher in einer intensiveren und breiteren Analyse der planungspraktischen Vorgehensweisen, ihrer (kulturellen) Einflussfaktoren und Steuerungsoptionen gesehen. Wünschenswert wäre es, ein breiteres Spektrum an kontextspezifischen Ansätzen und Interpretationen abzubilden. So können Akteuren und Akteursgruppen vor Ort innovative Ideen gezeigt werden, die für ihre spezifische Situation passfähig sind. Bisherige Veröffentlichungen zu integrierten Entwicklungen innerstädtischer Flusslandschaften beziehen sich entweder ausschließlich auf die Artefakte, also wie das „Design“ von integrierten Maßnahmen (Hochwasserschutz und Freiraumgestaltung beispielsweise) aussieht, oder, in der weit geringeren Anzahl der Fälle, auf das Prozessdesign. Umfangreiche Aussagen oder Good-practice-Beispiele, die sowohl Artefakte als auch den integrativen Prozess darstellen und in ihrem inhaltlichen Spektrum über die Passfähigkeit von (fach-) planerischen Instrumenten, kreativen Finanzierungsmöglichkeiten, synergienutzenden Designs bis hin zu Prozessgestaltungen reichen, wären eine wichtige Grundlage für die Qualifizierung integrierter Entwicklungskonzepte in innerstädtischen Flusslandschaften.

\section{Literatur}

Adelle, C.; Russel, D. (2013): Climate Policy Integration: a Case of Déjà Vu? In: Environmental Policy and Governance 23, 1, 1-12.

Albrecht, J.; Wendler, W. (2009): Koordinierte Anwendung von Wasserrahmenrichtlinie und Hochwasserrisikomanagementrichtlinie im Kontext des Planungsprozesses. In: Natur und Recht 31, 9, 608-618.

Bachmann, J.; Hage, G. (2012): Leitfaden für die kommunale Landschaftsplanung in Baden-Württemberg. Der Landschaftsplan im Detail. Karlsruhe. http://www4.lubw.badenwuerttemberg. de/servlet/is/41319/Landschaftsplan_im_Detail.pdf?command $=$ downloadContent\&filename $=$ Landschaftsplan_im_Detail.pdf (0.12.2015).

Beckmann, P.; Fürst, D.; Scholles, F. (2004): Das System der räumlichen Planung in Deutschland. In: Fürst, D.; Scholles, F. (Hrsg.): Handbuch Theorien und Methoden der Raum- und Umweltplanung. Dortmund, 36-53.

Benz, A.; Dose, N. (2010): Governance - Modebegriff oder nützliches sozialwissenschaftliches Konzept. In: Benz, A.; Dose, N. (Hrsg.): Governance - Regieren in komplexen Regelsystemen. Eine Einführung. Wiesbaden, 13-36. = Governance, 1.

Benz, A.; Lütz, S.; Schimank, U.; Simonis, G. (2007): Handbuch Governance: Theoretische Grundlagen und empirische Anwendungsfelder. Wiesbaden. 
Bogumil, J.; Jann, W. (2009): Verwaltung und Verwaltungswissenschaft in Deutschland. Einführung in die Verwaltungswissenschaft. Wiesbaden.

Corbin, J. (2006): Grounded Theory. In: Bohnsack, R.; Marotzki, W.; Meuser, M. (Hrsg.): Hauptbegriffe Qualitativer Sozialforschung. Opladen, Farmington Hills, 70-75.

DiGaetano, A.; Strom, E. (2003): Comparative Urban Governance: An Integrated Approach. Urban Affairs Review 38, 3, 356-395.

Driessen, P. P. J.; Dieperink, C.; van Laerhoven, F.; Runhaar, H. A. C.; Vermeulen, W. J. V. (2012): Towards a Conceptual Framework for The Study of Shifts in Modes of Environmental Governance Experiences From The Netherlands. In: Environmental Policy and Governance 22, 3, 143-160.

Evers, M.; Krause, K.-U. (2010): Ein integratives Planungsinstrument für das Hochwasserrisikomanagement. In: Raumforschung und Raumordnung 68, 5, 409-418.

Friedmann, J. (2005): Globalization and the emerging culture of planning. In: Progress in Planning 64, 3, 183-234.

Fürst, D. (2001): Regional governance - ein neues Paradigma der Regionalwissenschaften? Raumforschung und Raumordnung 59, $5 / 6,370-380$.

Fürst, D. (2007): Planungskultur. Auf dem Weg zu einem besseren Verständnis von Planungsprozessen? pnd Online, III/2007.

Fürst, D. (2014): Koordination und Führung in der Regionalplanung. Raumforschung und Raumordnung 72, 6, 451-462.

Gärditz, K. F. (2013): Ökologischer Gewässerschutz zwischen Wasserrecht und Naturschutzrecht. In: Natur und Recht 35, 9, 605-613.

Gleick, P. H. (2000): A Look at Twenty-first Century Water Resources Development. In: Water International 25, 1, 127-138.

Grett, H.-D. (2011): Synergien bei der Umsetzung der Europäischen Wasserrahmenrichtlinie und den EG-Richtlinien zum Naturschutz, Meeresschutz, Hochwasserschutz und Klimaschutz. In: Korrespondenz Wasserwirtschaft 4, 5, 252-257.

Haaren, C. von; Galler, C. (Hrsg.) (2011): Zukunftsfähiger Umgang mit Wasser im Raum. Hannover. $=$ Forschungs- und Sitzungsberichte der ARL, 234

Haaren, C. von; Galler, C. (2012): Landschaftsplanung. Grundlage nachhaltiger Landschaftsentwicklung. https://www.bfn.de/ fileadmin/MDB/documents/service/Landschaftsplanung_2012. pdf (04.12.2015).

Haaren, C. von; Moss, T. (2011): Voraussetzungen für ein integriertes Management: Koordination und Kooperation der wasserrelevanten Akteure und Organisationen in Deutschland. In: Haaren, C. von; Galler, C. (Hrsg.): Zukunftsfähiger Umgang mit Wasser im Raum. Hannover, 67-81. = Forschungs- und Sitzungsberichte der ARL, 234.

Hartmann, T. (2010): Reframing Polyrational Floodplains: Land Policy for Large Areas for Temporary Emergency Retention. In: Nature and Culture 5, 1, 15-30.

Hartmann, T.; Driessen, P. (2014): The flood risk management plan: towards spatial water governance. In: Journal of Flood Risk Management, Virtual Special Issue: The European Flood Risk Management Plan. DOI: 10.1111/jfr3.12077

Huitema, D.; Mostert, E.; Egas, W.; Moellenkamp, S.; Pahl-Wostl, C.; Yalcin, R. (2009): Adaptive water governance: assessing the institutional prescriptions of adaptive (co-) management from a governance perspective and defining a research agenda. In: Ecology and Society 14, 1, 26.

Hurck, R. (2011): Kostenträgerschaft und Finanzierung von Maßnahmen zur Gewässerentwicklung und des Hochwasserschutzes. In: Haaren, C. von; Galler, C. (Hrsg.): Zukunftsfähiger Umgang mit Wasser im Raum. Hannover, 104-109. = Forschungs- und Sitzungsberichte der ARL, 234.

Jänicke, M.; Volkery, A. (2005): Artikel „Umweltpolitik“. In: Ritter, E.-H.; Bröcker, J.; Fürst, D. et al. (Hrsg.): Handwörterbuch der Raumordnung. Hannover, 1179-1185.
Jordan, A.; Schout, A.; Unfried, M. (2013): Policy coordination. In: Jordan, A.; Adelle, C. (Hrsg.): Environmental policy in the EU. Actors, institutions and processes. London, New York, 227-246.

Kappet, J. (2006): Qualitätsorientierter Gewässerschutz in Deutschland. Baden-Baden. $=$ Forum Umweltrecht, 55.

Knüppe, K.; Pahl-Wostl, C. (2013): Requirements for adaptive governance of groundwater ecosystem services: insights from Sandveld (South Africa), Upper Guadiana (Spain) and Spree (Germany). In: Regional Environmental Change 13, 1, 53-66.

Köck, W. (2007): Fachplanung und Bodenschutz. Bestandsaufnahme und Reformüberlegungen. In: Köck, W.; Bovet, J.; Gawron, T.; Hofmann, E.; Möckel, S.: Effektivierung des raumbezogenen Planungsrechts zur Reduzierung der Flächeninanspruchnahme. Berlin, 165-184. = UBA-Bericht 01/07

Köck, W.; Bovet, J. (2015): Koordinierung der Flussgebietsbewirtschaftung - unter besonderer Berücksichtigung der Abstimmung mit der Raumordnung. In: Karl, H. (Hrsg.): Koordination raumwirksamer Politik. Mehr Effizienz und Wirksamkeit von Politik durch abgestimmte Arbeitsteilung. Hannover, 131-150. = Forschungsberichte der ARL, 4.

Laskowski, S. R.; Ziem, C. (2014): Gewässerschutzrecht. In: Koch, H. J. (Hrsg.): Umweltrecht. München, 296-358.

Levin-Keitel, M. (2014): Managing urban riverscapes: towards a cultural perspective of land and water governance. In: Water International 39, 6, 842-857.

Levin-Keitel, M. (2015): Einblicke in die empirische Erforschung von Planungskulturen - Flusslandschaften in der Stadt. pnd Online, im Erscheinen.

Levin-Keitel, M.; Sondermann, M. (2014): Planerische Instrumente in lokalen Kontexten. Einblicke in die Vielfalt von Planungskulturen. In: Grotheer, S.; Schwöbel, A.; Stepper, M. (Hrsg.): Nimm's sportlich - Planung als Hindernislauf. Hannover, 172-191. = Arbeitsberichte der ARL, 10.

Lüders, C. (2011): Teilnehmende Beobachtung. In: Bohnsack, R.; Marotzki, W.; Meuser, M. (Hrsg.): Hauptbegriffe Qualitativer Sozialforschung. Opladen, Farmington Hills, 151-153.

Maaß, C. A.; Schütte, P. (2014): Naturschutzrecht. In: Koch, H. J. (Hrsg.): Umweltrecht. München, 417-475.

Margerum, R. D. (2008): A Typology of Collaboration Efforts in Environmental Management. In: Environmental Management 41, 4, 487-500.

Marotzki, W. (2011): Leitfadeninterview. In: Bohnsack, R.; Marotzki, W.; Meuser, M. (Hrsg.): Hauptbegriffe Qualitativer Sozialforschung. Opladen, Farmington Hills, 114.

Meuser, M.; Nagel, U. (2011): Experteninterview. In: Bohnsack, R.; Marotzki, W.; Meuser, M. (Hrsg.): Hauptbegriffe Qualitativer Sozialforschung. Opladen, Farmington Hills, 57-58.

Molle, F. (2008): Nirvana concepts, narratives and policy models: Insight from the water sector. In: Water Alternatives 1, 1, 131-156.

Moss, T. (2009): Zwischen Ökologisierung des Gewässerschutzes und Kommerzialisierung der Wasserwirtschaft: Neue Handlungsanforderungen an Raumplanung und Regionalpolitik. Raumforschung und Raumordnung 67, 1, 54-68.

Moss, T.; Newig, J. (2010): Multilevel Water Governance and Problems of Scale: Setting the Stage for a Broader Debate. In: Environmental Management 46, 1, 1-6.

Nielsen, H. Ø.; Frederiksen, P.; Saarikoski, H.; Rytkönen, A.-M.; Pedersen, A. B. (2013): How different institutional arrangements promote integrated river basin management. Evidence from the Baltic Sea Region. In: Land Use Policy 30, 1, 437-445.

Nuissl, H.; Heinrichs, D. (2011): Fresh Wind or Hot Air - Does the Governance Discourse Have Something to Offer to Spatial Planning? Journal of Planning Education and Research 31, 1, $47-59$. 
Othengrafen, F. (2012): Uncovering the Unconscious Dimensions of Planning. Using Culture as a Tool to Analyse Spatial Planning Practice. Farnham.

Othengrafen, F.; Sondermann, M. (Hrsg.) (2015): Städtische Planungskulturen im Spiegel von Konflikten, Protesten und Initiativen. = Planungsrundschau, 23.

Paavola, J.; Gouldson, A.; Kluvánková-Oravská, T. (2009): Interplay of actors, scales, frameworks and regimes in the governance of biodiversity. In: Environmental Policy and Governance 19, 3, $148-158$.

Pahl-Wostl, C.; Lebel, L.; Knieper, C.; Nikitina, E. (2012): From applying panaceas to mastering complexity: Toward adaptive water governance in river basins. In: Environmental Science \& Policy 23, 24-34.

Poch, M.; Comas, J.; Rodríguez-Roda, I.; Sànchez-Marrè, M.; Cortés, U. (2004): Designing and building real environmental decision support systems. In: Environmental Modelling \& Software 19, 9, 857-873.

Ramsauer, U. (2014): Allgemeines Umweltverwaltungsrecht. In: Koch, H. J. (Hrsg.): Umweltrecht. München, 114-200.

Rauschmayer, F.; Berghöfer, A.; Omann, I.; Zikos, D. (2009): Examining processes or/and outcomes? Evaluation concepts in European governance of natural resources. In: Environmental Policy and Governance 19, 3, 159-173.

Reimer, M. (2012): Planungskultur im Wandel: das Beispiel der REGIONALE 2010. Detmold.

Reinhardt, M. (2009): Zum Verhältnis von Wasserrecht und Naturschutzrecht. In: Natur und Recht 31, 8, 517-525.

Sanden, J. (2014): Bodenschutz und Altlastenrecht. In: Koch, H. J. (Hrsg.): Umweltrecht. München, 476-525.

Sanon, S.; Hein, T.; Douven, W.; Winkler, P. (2012): Quantifying ecosystem service trade-offs: The case of an urban floodplain in Vienna, Austria. In: Journal of Environmental Management 111, $159-172$.

SRU - Sachverständigenrat für Umweltfragen (2004): Umweltpolitische Handlungsfähigkeit sichern. Umweltgutachten 2004. Baden-Baden.

SRU - Sachverständigenrat für Umweltfragen (2008): Umweltschutz im Zeichen des Klimawandels. Umweltgutachten 2008. Berlin.

Stadt Nürnberg (2012): Integriertes Stadtentwicklungskonzept Nürnberg am Wasser. Nürnberg.

Wasserwirtschaftsamt Regensburg; Stadt Regensburg (2010): Hochwasserschutz Stadt Regensburg. Flussraumkonzept Donau Regen. Regensburg.

Wiering, M.; Immink, I. (2006): When water management meets spatial planning: a policy-arrangements perspective. Environment and Planning C: Government and Policy 24, 3, 423-438.

\section{Verzeichnis der Rechtsgrundlagen}

Baugesetzbuch in der Fassung der Bekanntmachung vom 23. September 2004 (BGB1. I S. 2414), das durch Artikel 6 des Gesetzes vom 20. Oktober 2015 (BGBl. I S. 1722) geändert worden ist.

Bayerisches Wassergesetz (BayWG) vom 25.02.2010 (GVBl 2010, S. 66), berichtigt am 05.03.2010 (GVB1 2010, S. 130), zuletzt geändert am 16. Februar 2012 (GVBL 2012 Nr. 3, S. 40).

Bundes-Bodenschutzgesetz vom 17. März 1998 (BGBl. I S. 502), das zuletzt durch Artikel 101 der Verordnung vom 31. August 2015 (BGB1. I S. 1474) geändert worden ist.

Bundesnaturschutzgesetz vom 29. Juli 2009 (BGB1. I S. 2542), das zuletzt durch Artikel 421 der Verordnung vom 31. August 2015 (BGBl. I S. 1474) geändert worden ist.

EU-Wasserrahmenrichtlinie (2000/60/EG): Richtlinie 2000/60/EG des Europäischen Parlaments und des Rates vom 23. Oktober 2000 zur Schaffung eines Ordnungsrahmens für Maßnahmen der Gemeinschaft im Bereich der Wasserpolitik (AB1. Nr. L 327 vom 22/12/2000 S. 0001-0073).

Grundgesetz für die Bundesrepublik Deutschland in der im Bundesgesetzblatt Teil III, Gliederungsnummer 100-1, veröffentlichten bereinigten Fassung, das zuletzt durch Artikel 1 des Gesetzes vom 23. Dezember 2014 (BGB1.I S. 2438) geändert worden ist.

Raumordnungsgesetz vom 22. Dezember 2008 (BGB1. I S. 2986), das zuletzt durch Artikel 124 der Verordnung vom 31. August 2015 (BGB1. I S. 1474) geändert worden ist.

Wasserhaushaltsgesetz vom 31. Juli 2009 (BGB1. I S. 2585), das zuletzt durch Artikel 320 der Verordnung vom 31. August 2015 (BGB1. I S. 1474) geändert worden ist.

\section{Verzeichnis der Interviews}

BY_N_S1, BY_N_S2, BY_N_S3, BY_N_S4: Vertreter der Stadtverwaltung in Nürnberg, Bayern

BY_N_W1: Vertreter der Wasserwirtschaft in Nürnberg, Bayern

BY_R_S1, BY_R_S2: Vertreter der Stadtverwaltung in Regensburg, Bayern

BY_R_W1, BY_R_W2, BY_R_W3, BY_R_W4: Vertreter der Wasserwirtschaft in Regensburg, Bayern

NI_A1, NI_A2, NI_A3: Vertreter der Kommunalverwaltung in Niedersachsen

NI_B1, NI_B2: Vertreter der Landesverwaltung Niedersachsen (Niedersächsischer Landesbetrieb für Wasserwirtschaft, Küstenund Naturschutz)

NI_C1, NI_C2, NI_C3: Vertreter der Landesverwaltung Niedersachsen (Niedersächsisches Ministerium für Umwelt, Energie und Klimaschutz, Niedersächsisches Ministerium für Ernährung, Landwirtschaft und Verbraucherschutz) 